\title{
A Circular Pneumatic Muscle Actuator (CPMA) Inspired by Human Skeletal Muscles
}

\author{
Alaa Al-Ibadi, Samia Nefti-Meziani, and Steve Davis
}

\begin{abstract}
This paper illustrates the design, implementation and kinematics of a novel circular pneumatic muscle actuator (CPMA), inspired by the skeletal muscles of a human. The variation of the inner diameter of this actuator is a unique feature. Furthermore, CPMA produces a radial force towards its centre by increasing the diameter of the actuator itself in addition to the reduction in the inner diameter. These performances make the presented actuator suitable to use in numerous applications. The grasping by a soft gripper is chosen as an application to design an efficient soft gripper by using single and multiple CPMAs.
\end{abstract}

Keywords- Circle Pneumatic Muscle Actuator (CPMA); Human Skeletal Muscles; Kinematics; Human Inspiration; Soft Grippers.

\section{INTRODUCTION}

Biological inspiration provides numerous artificial benefits to our lives, such as a pneumatic muscle actuator (PMA), which was presented by Joseph L. McKibben in the 1950 's and is built from an inner rubber tube bound by a braided sleeve [1]. The construction of the PMA defines the type of actuator. By selecting the length of both the inner tube and the braided sleeve, the PMA acts as a contraction actuator if the braided angle $(\theta)$, which is measured between the braided stand and the vertical line, is less than $54.7^{0}$ [2] [3], and as an extension actuator if the angle is more than $54.7^{0}$ [4]. Fig. 1 shows the braided angle of the PMA for both the contractor and the extensor actuators. Moreover, the PMA will produce a tensile force for the contractor type and an extension force for the extensor PMA. The force value for both types depends on the structure and the material specifications of the PMA [1] [5]

It is primarily over the last few decades that researchers have developed soft actuators. As a result, soft robotics have been extensively used in numerous areas. Researchers from different fields, such as material science, physics, biology, mechanical and control engineering, have a big interest in such types of robots [6]. This developing research field focuses on robots made of soft materials, which increases

Alaa Al-Ibadi is with the Computing, Science and Engineering, Autonomous Systems and Robotics research centre, University of Salford, Manchester, UK and Computer Engineering Department, University of Basrah, Iraq(email:a.f.a.al-ibadi@edu.salford.ac.uk, alaa.falah77@ieee.org), (+44(0)7438462585).

Samia Nefti-Meziani and Steve Davis are with the Computing, Science and Engineering, Autonomous Systems and Robotics research centre, University of Salford, Manchester, UK (email: S.NeftiMeziani@salford.ac.uk), (email: S.T.Davis@salford.ac.uk). the safety of human-robot interaction, and makes the robot more compliant in its environment [7] [8]. An infinite number of degrees of freedom (DOF) is achievable with soft robots due to the bending capability of the soft actuator [9]. Soft robots have an advantage over rigid robots, as they produce only a slight resistance to obstacles and can adapt to them. Consequently, soft and fragile payloads could be handled without causing any damage [10].

Important research has been done on the design, implementation and modelling of the soft actuators and continuum arms. Among the improvements made is the PneuNet actuator by Ilievski, et al. [11]. The authors present a bending actuator by changing the wall thickness. This actuator is easy to manufacture but the ratio of the elasticity that can be achieved is too limited. To overcome this limitation, Deimel and Brock [12] used a PneuFlex actuator which implants polymer fibres to reinforce the rubber substrate. The elasticity of the polyethylene terephthalate (PET) material is three to four times less than silicon. AlIbadi, et al. [13] designed a self-bending contraction actuator (SBCA) by inserting a reinforced flexible rod to one side of the contraction PMA to achieve a bending behaviour. AlFahaam, et al. [14] proposed a bending behaviour by modifying the structure of the extensor PMA by using a high-tension thread to reinforce one side of the covered sleeve.

Faudzi, et al. [15] used the efficiency of the braided angle on the actuators performance to design a bending behaviour by using two different braided sleeves of different braided angles to cover the inner tube of the contractor PMA.

In this paper, a novel design of a circular pneumatic muscle actuator (CPMA) has been proposed. The kinematics of the new actuator is presented then a grasping application is suggested to validate the performance of the CPMA.

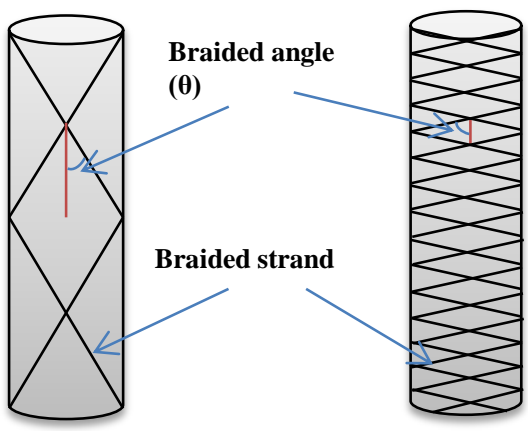

(a)

(b)

Figure 1. The braided angle of the PMA. (a) Contraction actuator. (b) Extension actuator. 


\section{Human FACIAL MUSCLES}

Human facial muscles have unique features. They lie on the top of the body joints and their function is either to open and close the orifices of the face, or to pull the skin into intricate actions, creating facial expressions.

There is little information about the structure and performance of the facial muscles in comparison to other skeletal muscles in the body [16].

Martini, et al. [17] argue that muscle tissues share four basic properties:

1- Excitability: the ability to respond to stimulation

- Skeletal muscles normally respond to stimulation via the nervous system.

- Cardiac and smooth muscles respond to the nervous system and circulating hormones.

2- Contractility: the ability to actively shorten and exert a pull or tension that can be harnessed by connective tissues.

3- Extensibility: the ability to continue to contract over a range of resting lengths.

4- Elasticity: the ability of a muscle to rebound toward its original length after a contraction.

The circular "Orbicularis Oculi" muscle controls the movements of both the mouth and the eyes. Fig. 2 shows the shape of the human circular muscle. The contraction of this muscle decreases the mouth slot, while the resting causes the mouth to open. Similar effects occur in the human eyes.

This singular type of human skeletal muscle inspired us to design the CPMA, which has an ability to decrease its inner area by shrinking the outer and inner circumference and increase its diameter.

\section{THE DESIGN OF THE CPMA}

The way to build a contraction PMA is also used to design and implement the CPMA. Similar lengths of a braided sleeve of 2.5 to $4.5 \mathrm{~cm}$ diameter variation and a rubber tube of $1.1 \mathrm{~cm}$ diameter are used to build the CPMA.

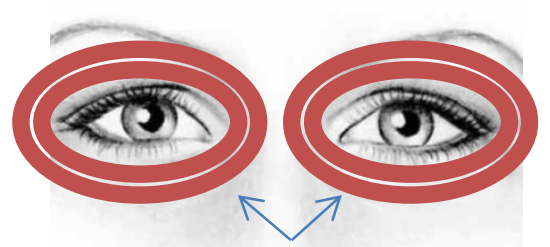

Eyes circular muscles

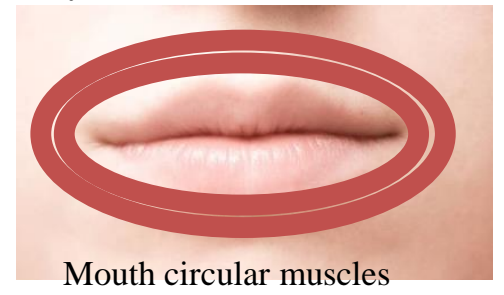

Figure 2. The circular muscle of the eyes and the mouth of a human.
The two ends are connected together by a $5 \mathrm{~cm}$ aluminium cylinder.

By pressurising the actuator, both the outer and the inner diameter of the CPMA will reduce, while the diameter of the actuator itself will increase until the braided angle reaches its critical value or the maximum value of the sleeve diameter is achieved. The triple diameter changes lead to a decrease in the opening area.

The opening area at relaxed condition (zero air pressure) depends on the rest length of the braided sleeve and its diameter.

\section{THE KINEMATICS OF THE CPMA}

Fig. 3.a-b illustrates the geometrical analysis of the PMA and the contractor actuator of length $\mathrm{L}$ and diameter $\mathrm{D}$. By bending this actuator until the two ends connect to each other, the resulting shape is two overlapping circles as shown in Fig.3.c.
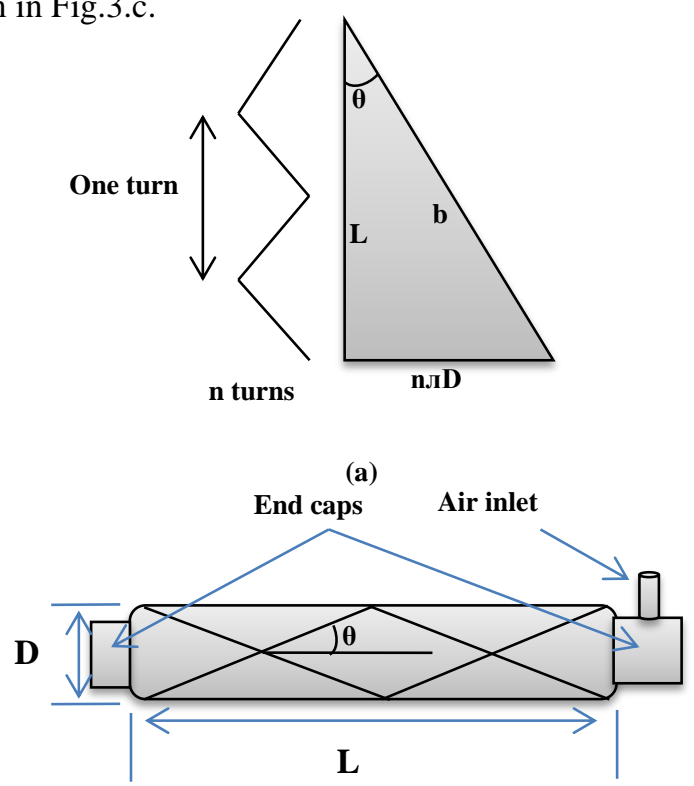

(b)

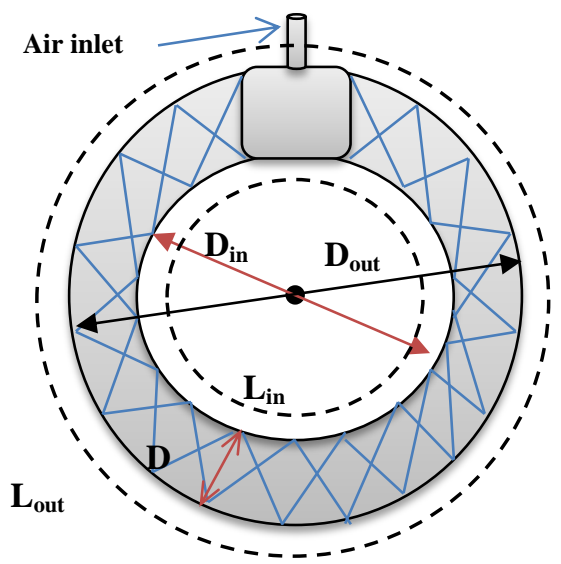

(c)

Figure 3. The geometrical structure of the CPMA. (a) the geometrical of the PMA. (b) The contraction actuator. (c) The circular actuator. 
From Fig. 3.a:

$$
\tan \theta=\frac{n \pi D}{L}
$$

Where $\theta$ is a braided angle and $n$ is the turn numbers of strand length $b$.

Assuming that the diameter $D$ of the contraction actuator in Fig. 3.b is equal to the diameter of the CPMA and from Fig. 3.c:

$$
D_{\text {in }}=D_{\text {out }}-2 D
$$

Where $D_{\text {in }}$ is the inner diameter of CPMA and $D_{\text {out }}$ is the outer diameter. The outer circumference $\left(L_{\text {out }}\right)$ and the inner circumference $\left(L_{i n}\right)$ of the CPMA are defined in (3) and (4) as follows:

$$
\begin{aligned}
& L_{\text {out }}=\pi D_{\text {out }} \\
& L_{\text {in }}=\pi D_{\text {in }}
\end{aligned}
$$

Assuming:

$$
L_{\text {out }}=L
$$

By substituting (5) in (3):

$$
D_{\text {out }}=\frac{L}{\pi}
$$

By substituting (1) and (6) in (2):

$$
D_{i n}=\frac{L}{\pi}-2 \frac{L \tan \theta}{n \pi}
$$

Or:

$$
D_{\text {in }}=\frac{L}{\pi}\left(1-2 \frac{\tan \theta}{n}\right)
$$

From (4):

$$
L_{\text {in }}=L\left(1-2 \frac{\tan \theta}{n}\right)
$$

The contraction ratio for the contractor PMA is defined by:

$$
\varepsilon=\frac{L_{0}-L}{L_{0}}
$$

Or:

$$
L=L_{0}(1-\varepsilon)
$$

Where: $L_{0}$ is the length of the contraction actuator in the relaxed condition. By substituting (11) in (9):

$$
L_{\text {in }}=L_{0}(1-\varepsilon)\left(1-2 \frac{\tan \theta}{n}\right)
$$

From (8) and (12) the inner diameter and the inner circumference of the CPMA can be determined from the geometrical analysis of the contraction actuator.

\section{THE RADIAL FORCE OF THE CPMA}

At pressurised conditions, the CPMA applied a force towards the centre of the actuator, along the radius. This force is created due to the shortness of the inner diameter.

By applying a virtual work law, the derivative of the input work $\left(W_{i n}\right)$ of the actuator is:

$$
d W_{i n}=p \cdot d V
$$

Where: $p$ is the actuator pressure in $(\mathrm{kPa})$ and $V$ is the actuator volume in $\left(\mathrm{m}^{3}\right)$. Fig. 4 shows the CPMA as a cylinder. The base area is a circular diameter $D_{\text {in }}$ and the height is the actuator diameter $D$.

Both the inner diameter and the actuator diameter are changing with pressure which causes force $(F)$ towards the centre, and the derivative of the output work $\left(W_{\text {out }}\right)$ is:

$$
d W_{\text {out }}=F \cdot d D
$$

Assuming that the losses are zero, the change in input work is equal to the change in output work:

$$
d W_{\text {in }}=d W_{\text {out }}
$$

Or:

$$
F=p \frac{d V}{d D}
$$

The volume of the cylinder $V$ equal to:

$$
V=\frac{\pi D_{i n}^{2} D}{4}
$$

From (1) the height of the cylinder is:

$$
D=\frac{L \tan \theta}{n \pi}
$$

Substituting (7) and (18) in (17): 


\section{$\mathrm{D}_{\text {in }}$}

$\mathrm{D}$

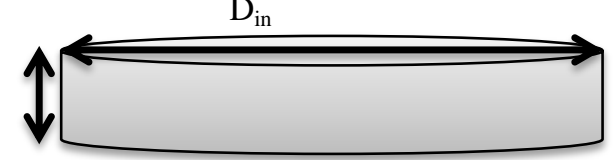

Figure 4. The geometrical structure of the CPMA as a cylinder.

$$
V=\frac{1}{4}\left(\frac{L}{\pi}\left(1-2 \frac{\tan \theta}{n}\right)\right)^{2}\left(\frac{L \tan \theta}{n}\right)
$$

Differentiation in both the volume and the height with respect to $\theta$ gives:

$$
\frac{d V}{d D}=\frac{d V}{d \theta} \times \frac{d \theta}{d D}
$$

Then:

$$
\frac{d V}{d \theta}=\frac{L}{n \pi \cos ^{2} \theta}\left(\frac{3 L^{2} \tan ^{2} \theta}{n^{2} \pi}-\frac{2 L^{2} \tan \theta}{n \pi}+\frac{L^{2}}{4 \pi}\right)
$$

And:

$$
\frac{d D}{d \theta}=\frac{L}{n \pi \cos ^{2} \theta}
$$

Substituting (21) and (22) in (20) gives:

$$
\frac{d V}{d D}=\frac{3 L^{2} \tan ^{2} \theta}{n^{2} \pi}-\frac{2 L^{2} \tan \theta}{n \pi}+\frac{L^{2}}{4 \pi}
$$

The force in (16) can be found as follows:

$$
F=p \frac{L^{2}}{4 n^{2} \pi}\left(12 \tan ^{2} \theta-8 n \tan \theta+n^{2}\right)
$$

Since the effective circumference is less than the actual length due to the length of the solid cylinder, then:

$$
L=L+L_{s}
$$

Where: $\dot{L}$ is the effective outer circumference and $L_{s}$ is length of the solid cylinder. Furthermore, assuming the required pressure to get a contact between the inner tube and the braided sleeve is $p_{0}$, then:

$$
F=\left(p-p_{0}\right) \frac{L^{2}}{4 n^{2} \pi}\left(12 \tan ^{2} \theta-8 n \tan \theta+n^{2}\right)
$$

\section{DESIGN A SOFT GRIPPER}

A novel design is proposed in this section by using the extensor PMA and the CPMA. Fig. 5 explains the structure of this gripper, which is built using three $18 \mathrm{~cm}$ extensor actuators and one CPMA.

The extensor actuators provide an ability to extend and bend in addition to increasing the gripper's stability, while the grasping occurs due to the CPMA, which is made at a 30 $\mathrm{cm}$ relaxed length. The maximum inner diameter of the gripper is $7.8 \mathrm{~cm}$.
Experiments have been done to define the performance of the proposed gripper. Air pressure is applied by using a solenoid valve to the extensor actuators, which changes the length of the gripper. The length of the gripper changes with pressure until it reaches the maximum length of $24 \mathrm{~cm}$ at $500 \mathrm{kPa}$ with an extension ratio of $33 \%$. Then pressure is applied to the CPMA and the inner diameter is reduced to the minimum of $4.45 \mathrm{~cm}$ at $400 \mathrm{kPa}$. Fig. 6 shows the diameter and the length as a function of pressure. Further air pressure is added to the contractor ring but the inner diameter remains constant because the contractor muscle reaches its maximum contraction ratio. Therefore, the percentage of the diameter reduction after the $400 \mathrm{kPa}$ can be ignored.

The diameter reduction ratio $(D R R)$ can be calculated from (27) and it is equal to $43 \%$ for the presented gripper.

$$
D R R=\frac{D_{\text {in } 0}-D_{\text {in }}}{D_{\text {in } 0}}
$$

Where: $D_{\text {in } 0}$ is the inner diameter at zero pressure and $D_{\text {in }}$ is the inner diameter of the pressurised conditions.

This gripper provides an infinite number of contact points between the inner surface of the CPMA and the object to be handled. This preference increases the applied force and provides a significant grasping stability. On the other hand, pressurising the extensor PMAs simultaneously results in increasing the gripper length, as shown in Fig.6, while different pressure amounts in each actuator lead to moving the ring in multiple directions. The maximum angle is $61^{\circ}$ in relation to its original position and can be achieved by applying air pressure to one actuator at no load. These performances increase the efficiency of the gripper by adding the bending behaviour.

To explain the pressure-payload characteristic for this gripper, an experiment has been done by selecting multiweight cylindrical objects of $6 \mathrm{~cm}$ diameter. The load starts at $0.5 \mathrm{~kg}$ and is then increased by $0.5 \mathrm{~kg}$ steps. At each step, the applied air pressure is raised to prevent slipping. Fig. 7 illustrates the experimental results and shows that the maximum payload for the presented gripper is $10.9 \mathrm{~kg}$ for the $6 \mathrm{~cm}$ object and the payload-pressure characteristic is linear above the $1.5 \mathrm{~kg}$ load. The parameter to be controlled for the presented gripper is the air pressure in the CPMA, which provides an easy strategy for achieving the grasping operation.

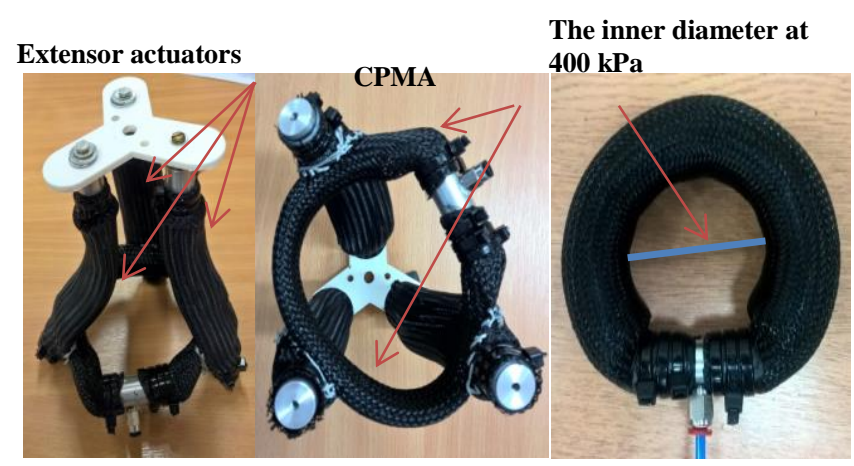

Figure 5. The structure of the extension-CPMA gripper. 


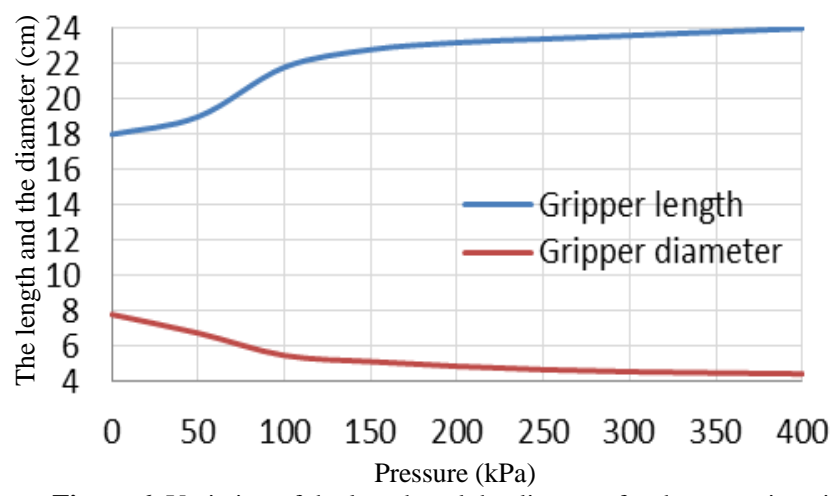

Figure 6. Variation of the length and the diameter for the extension-ring gripper

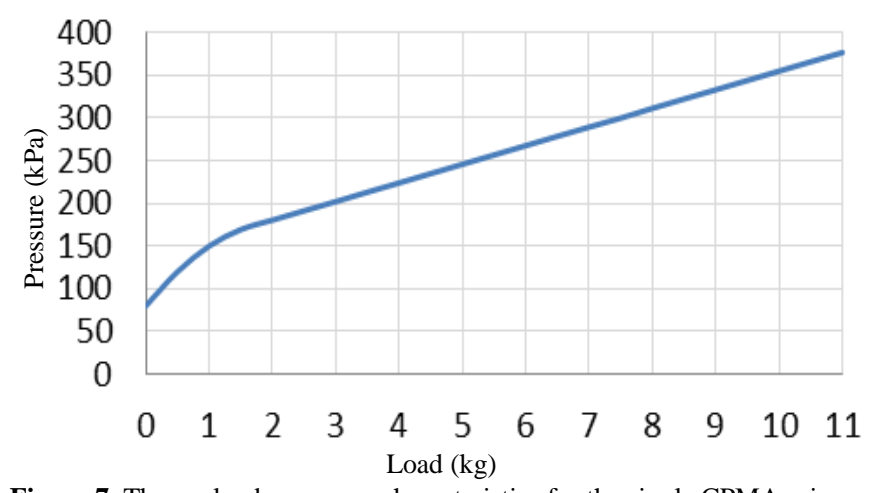

Figure 7. The payload -pressure characteristics for the single CPMA gripper.

Objects of various shapes can be grasped, however, their size has to be limited to no more than $3.9 \mathrm{~cm}$ between the object's centre and its edge. Fig. 8 shows the grasping of different objects using the proposed gripper. Different object shapes and weights require a different grasping force. However, the proposed gripper provides equal grasping force for all contact points between the objects and the CPMA.

\section{THREE CPMAS GRIPPER}

In this section, the proposed gripper is redesigned by increasing the number of the CPMAs to three in order to increase the grasping payload. In this design, the length of the gripper at zero pressure is $27 \mathrm{~cm}$ and it is increased to $38.1 \mathrm{~cm}$ at $500 \mathrm{kPa}$. The extension ratio for the extensor muscles is $41 \%$. The diameter of the CPMA varies from 8 $\mathrm{cm}$ to $4.3 \mathrm{~cm}$ for the maximum pressure of $400 \mathrm{kPa}$. The diameter reduction ratio for these rings is $46 \%$. Fig. 10 illustrates the three-ring gripper and its performances, which are illustrated in Fig. 11.

A similar experiment in section VI is used to define the grasping load of the three-ring gripper. For a cylindrical object with a $6 \mathrm{~cm}$ diameter, the gripper can grasp up to 40 $\mathrm{kg}$ while its weight is $0.8 \mathrm{~kg}$.

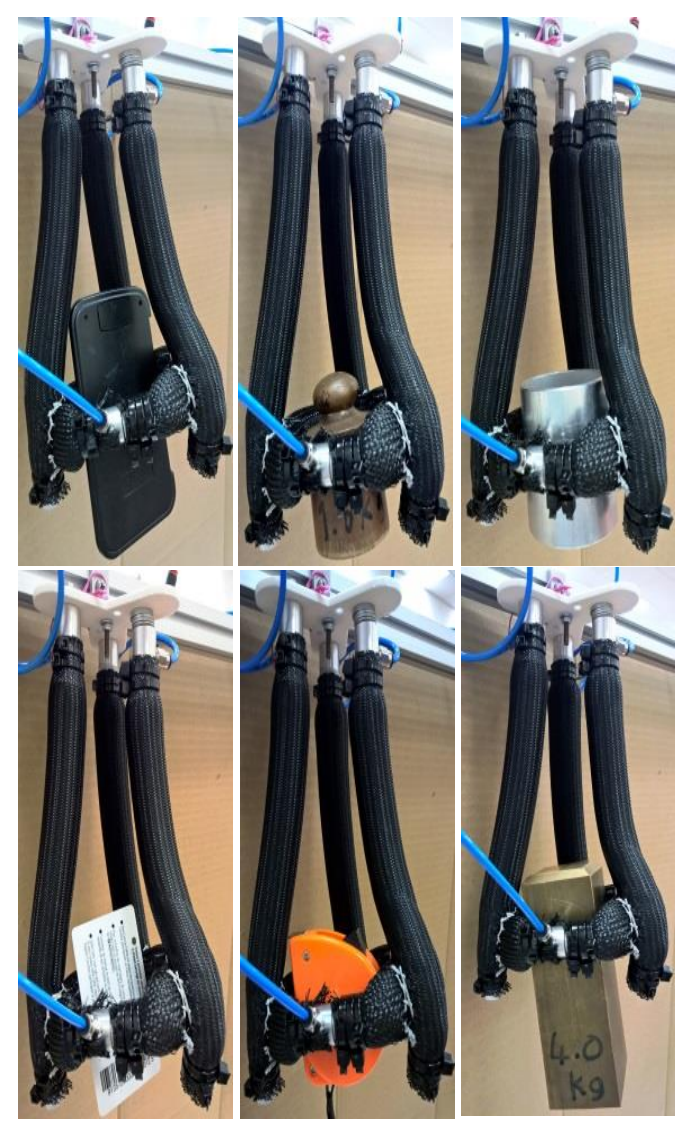

Figure 8. Multiple objects grasped by the extension-ring gripper.

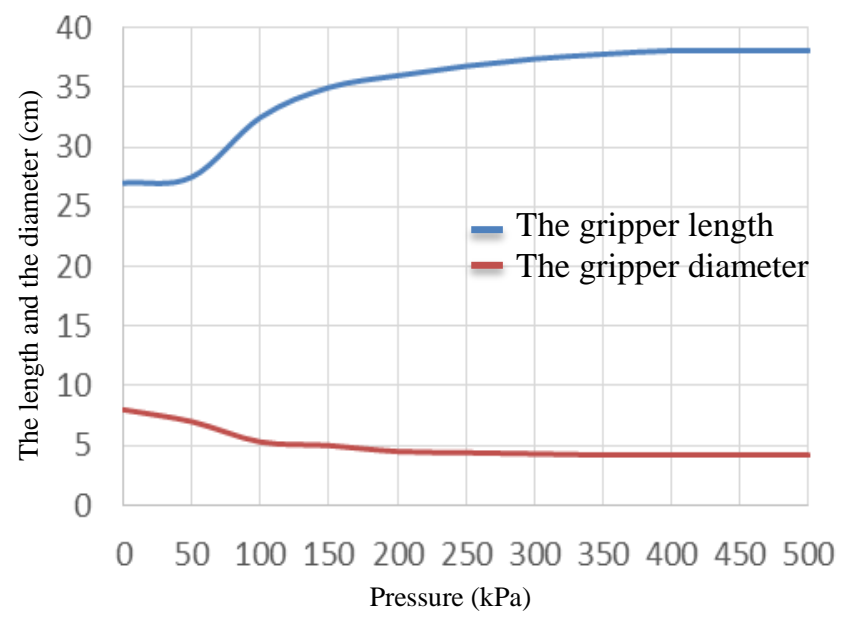

Figure 9. Variation of the length and the diameter for the three ring gripper. 


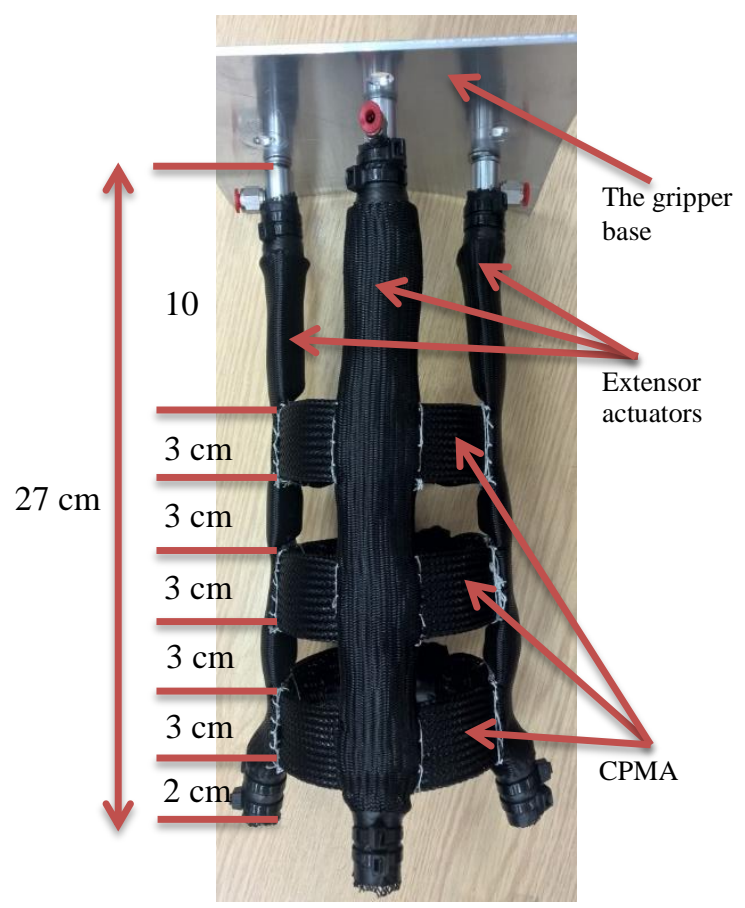

Figure 10. The dimensions and the structure of the three CPMA gripper.

\section{CONCLUSION}

A novel circular pneumatic muscle actuator (CPMA) inspired by human skeletal muscles is presented in this paper. The construction of the CPMA and its kinematics are proposed to explain the performance of the actuator.

The actuator produces a force towards its centre. The force is formulated with respect to the outer circumference and the braided angle.

To validate the proposed actuator, two grippers of one and three CPMAs are designed. The soft gripper shows that the single CPMA produces a grasping force of up to $10.9 \mathrm{~kg}$ for a selected cylinder object, and the three-CPMA gripper applies a grasping force of $40 \mathrm{~kg}$.

In the future, a control system could be designed to control the grasping force of the presented actuator.

\section{ACKNOWLEDGMENT}

The authors would like to thank the Ministry of Higher Education in Iraq, as well as the University of Basrah and its computer-engineering department, for providing scholarship support to the first author of this paper.

\section{REFERENCES}

[1] C.-P. Chou and B. Hannaford, "Measurement and modeling of McKibben pneumatic artificial muscles," IEEE Transactions on Robotics and Automation., vol. 12, pp. 90-102, 1996.
[2] E. Kelasidi, G. Andrikopoulos, G. Nikolakopoulos, and S. Manesis, "A survey on pneumatic muscle actuators modeling," in 2011 IEEE International Symposium on Industrial Electronics (ISIE), , 2011, pp. 1263-1269.

[3] A. Al-Ibadi, S. Nefti-Meziani, and S. Davis, "Valuable experimental model of contraction pneumatic muscle actuator," in 201621 st IEEE International Conference on Methods and Models in Automation and Robotics (MMAR), , Poland, 2016, pp. 744-749.

[4] A. Al-Ibadi, S. Nefti-Meziani, and S. Davis, "Novel models for the extension pneumatic muscle actuator performances," in 2017 23rd International Conference on Automation and Computing (ICAC), 2017, pp. 1-6.

[5] B. Tondu and P. Lopez, "Modeling and control of McKibben artificial muscle robot actuators," Control Systems, IEEE, vol. 20, pp. 15-38, 2000.

[6] L. Margheri and B. Trimmer, "Soft Robotics Community Events: Meeting Different Backgrounds for Common Challenges," Soft Robotics, vol. 1, pp. 236-238, 2014

[7] R. Mutlu, S. K. Yildiz, G. Alici, M. in het Panhuis, and G. M. Spinks, "Mechanical stiffness augmentation of a 3D printed soft prosthetic finger," in 2016 IEEE International Conference on Advanced Intelligent Mechatronics (AIM), 2016, pp. 7-12.

[8] T. E. Pillsbury, Q. Guan, and N. M. Wereley, "Comparison of contractile and extensile pneumatic artificial muscles," in 2016 IEEE International Conference on Advanced Intelligent Mechatronics (AIM), 2016, pp. 94-99.

[9] D. Trivedi, C. D. Rahn, W. M. Kier, and I. D. Walker, "Soft robotics: Biological inspiration, state of the art, and future research," Applied Bionics and Biomechanics, vol. 5, pp. 99-117, 2008.

[10] B. A. Jones and I. D. Walker, "Kinematics for multisection continuum robots," IEEE Transactions on Robotics, vol. 22, pp. 43-55, 2006.

[11] F. Ilievski, A. D. Mazzeo, R. F. Shepherd, X. Chen, and G. M. Whitesides, "Soft robotics for chemists," Angewandte Chemie, vol. 123, pp. 1930-1935, 2011.

[12] R. Deimel and O. Brock, "A compliant hand based on a novel pneumatic actuator," in Robotics and Automation (ICRA), 2013 IEEE International Conference on, 2013, pp. 2047-2053.

[13] A. Al-Ibadi, S. Nefti-Meziani, and S. Davis, "Cooperative project by self-bending continuum arms," in 2017 23rd International Conference on Automation and Computing (ICAC), Huddersfield, UK, 2017, pp. $1-6$.

[14] H. Al-Fahaam, S. Davis, and S. Nefti-Meziani, "The design and mathematical modelling of novel extensor bending pneumatic artificial muscles (EBPAMs) for soft exoskeletons," Robotics and Autonomous Systems, vol. 99, pp. 63-74, 2018.

[15] A. A. M. Faudzi, M. R. M. Razif, I. N. A. M. Nordin, K. Suzumori, S. Wakimoto, and D. Hirooka, "Development of bending soft actuator with different braided angles," in 2012 IEEE/ASME International Conference on Advanced Intelligent Mechatronics (AIM), , 2012, pp. 1093-1098.

[16] C. W. Goodmurphy and W. K. Ovalle, "Morphological study of two human facial muscles: orbicularis oculi and corrugator supercilii," Clinical Anatomy, vol. 12, pp. 1-11, 1999.

[17] F. Martini, M. J. Timmons, and R. B. Tallitsch, Human anatomy, 6th ed.: San Francisco: Pearson Benjamin Cummings, 2008. 